\title{
Marked abnormalities of plasma protein biomarkers in Creutzfeldt-Jakob disease (CJD)
}

\section{Simon Mead}

Plasma neurofilament light and total tau show marked elevations in human prion disease patients with subtype correlations and prognostic value.

Neurologists are fortunate to have splendid diagnostic tests for prion diseases. The CSF real-time quaking induced conversion assay (RT-QuIC test), in which abnormal prion protein stimulates the misfolding of recombinant protein, has proven to be sensitive and extremely specific for CJD. MRI features too, particularly high signal from the cortex, striatum or thalamus on diffusion weighted imaging, are highly characteristic. Why then is further biomarker research necessary?

Patients with CJD are too often diagnosed late in their clinical course when they can no longer contribute to plans for the end of their life, or participate in clinical trials. As the annual incidence of sporadic CJD in many countries is substantially higher than a decade ago, it seems likely that patients were (and maybe are) not diagnosed at all. Some delay and misdiagnosis likely relate to the specialist nature and accessibility of diagnostic tests - you have to think about CJD in the first place to prompt a request for the RT-QuIC assay. Additionally, whilst we have classifications systems for use at post-mortem, we don't have accurate methods to prognosticate in life. Prion disease clinical trials have lacked quantitative biomarkers of neurodegeneration. Abu-Rumeileh et al.'s large series of plasma and CSF NfL and t-tau protein biomarkers is therefore timely and welcome ${ }^{1}$.

Previously, preliminary reports have documented the elevation of proteins that leak into the plasma as the brain rapidly degenerates in $\mathrm{CJD}^{23}$. The new study confirms that plasma NfL and particularly ttau have value as diagnostic biomarkers, in comparison with other rapidly progressive dementias. Although the performance of plasma t-tau was slightly better than $\mathrm{NfL}$, it was not quite as good as $\mathrm{t}$ tau in CSF. A special strength of the study is post-mortem examination in a large proportion and classification using a widely adopted system based on prion protein types and histopathology, pioneered by the group. Plasma t-tau appeared to show marked differences between CJD subtypes, being exceptionally elevated in the common $\mathrm{MM}(\mathrm{V}) 1$ subtype of $\mathrm{CJD}$, in keeping with the extreme rates of progression of this subtype. In different ways, the two proteins could contribute to subtype diagnosis in life and better prognostication.

In CJD, it seems likely that these blood biomarkers will become increasingly relevant as they move from research to clinical service labs. There is potential for a triage test to identify the possibility of CJD or another rapidly progressive dementia and prompt rapid use of specialist, more specific testing. Clinical trials in human prion disease and prognostic modelling should certainly plan to include these assays, although longitudinal data and correlation with measures of disease severity are needed. For those at-risk who carry prion protein gene mutations, the extraordinary high values recorded in some symptomatic inherited prion diseases provoke the question of whether there are changes prior to clinical onset that would benefit preventive medicine ${ }^{4}$.

1. Abu-Rumeileh. Comparison between plasma and cerebrospinal fluid biomarkers for the early diagnosis and association with survival in prion disease. JNNP 2020

2. Thompson AGB, Luk C, Heslegrave AJ, et al. Neurofilament light chain and tau concentrations are markedly increased in the serum of patients with sporadic Creutzfeldt-Jakob disease, and 
tau correlates with rate of disease progression. J Neurol Neurosurg Psychiatry

2018;89(9):955-61. doi: 10.1136/jnnp-2017-317793 [published Online First: 2018/03/01]

3. Kovacs GG, Andreasson U, Liman V, et al. Plasma and cerebrospinal fluid tau and neurofilament concentrations in rapidly progressive neurological syndromes: a neuropathology-based cohort. Eur J Neurol 2017;24(11):1326-e77. doi: 10.1111/ene.13389 [published Online First: 2017/08/18]

4. Vallabh SM, Minikel EV, Williams VJ, et al. Cerebrospinal fluid and plasma biomarkers in individuals at risk for genetic prion disease. BMC Med 2020;18(1):140. doi: 10.1186/s12916020-01608-8 [published Online First: 2020/06/20] 